\title{
Truncation Dimension for Function Approximation
}

\author{
Peter Kritzer*, Friedrich Pillichshammer†, and G.W. Wasilkowski
}

\begin{abstract}
We consider approximation of functions of $s$ variables, where $s$ is very large or infinite, that belong to weighted anchored spaces. We study when such functions can be approximated by algorithms designed for functions with only very small number $\operatorname{dim}^{\operatorname{trnc}}(\varepsilon)$ of variables. Here $\varepsilon$ is the error demand and we refer to $\operatorname{dim}^{\operatorname{trnc}}(\varepsilon)$ as the $\varepsilon$-truncation dimension. We show that for sufficiently fast decaying product weights and modest error demand (up to about $\varepsilon \approx 10^{-5}$ ) the truncation dimension is surprisingly very small.
\end{abstract}

\section{Introduction}

In this paper, we consider weighted anchored spaces of $s$-variate functions with bounded (in $L_{p}$ norm, $\left.1 \leq p \leq \infty\right)$ mixed partial derivatives of order one. More precisely, the functions being approximated are from the Banach space $F_{s, p, \gamma}$ whose norm is given by

$$
\|f\|_{F_{s, p, \gamma}}=\left(\sum_{\mathfrak{u}} \gamma_{\mathfrak{u}}^{-p} \int_{[0,1]^{|\mathfrak{u}|}}\left|f^{(\mathfrak{u})}\left(\left[\boldsymbol{x}_{\mathfrak{u}} ; \mathbf{0}_{-\mathfrak{u}}\right]\right)\right|^{p} \mathrm{~d} \boldsymbol{x}_{\mathfrak{u}}\right)^{1 / p} .
$$

Here, the summation is with respect to the subsets $\mathfrak{u}$ of $[s]=\{1, \ldots, s\}$ (when $s=\infty$ the summation is with respect to all finite subsets of $\mathbb{N})$, and $f^{(\mathfrak{u})}\left(\left[\boldsymbol{x}_{\mathfrak{u}} ; \mathbf{0}_{-\mathfrak{u}}\right]\right)$ denotes the mixed partial derivatives $\prod_{j \in \mathfrak{u}} \frac{\partial}{\partial x_{j}}$ of $f$ with values of $x_{j}$ for $j \notin \mathfrak{u}$ being zero. A crucial role is played by the weights $\gamma_{\mathfrak{u}}$, which are non-negative real numbers that quantify the importance of sets $\boldsymbol{x}_{\mathfrak{u}}=\left(x_{j}\right)_{j \in \mathfrak{u}}$ of variables.

We continue our considerations from [5], where we dealt with low truncation dimension for numerical integration. We are interested in a very large number $s$ of variables including $s=\infty$. Similar to [5], by $\varepsilon$-truncation dimension (or truncation dimension for short) we mean (roughly) the smallest number $k$ such that the worst case error (measured in the $L_{q}$ space) of approximating $s$-variate functions $f\left(x_{1}, \ldots, x_{s}\right)$ by $f_{k}=f\left(x_{1}, \ldots, x_{k}, 0, \ldots, 0\right)$ is no greater than the error demand $\varepsilon$ (see Definition 1 for more). We denote this minimal number $k$ by $\operatorname{dim}^{\operatorname{trnc}}(\varepsilon)$.

Note that if the truncation dimension is small, say $\operatorname{dim}^{\operatorname{trnc}}(\varepsilon)=3$, then the $s$-variate approximation problem can be replaced by the much easier $\operatorname{dim}^{\text {trnc }}(\varepsilon)$-variate one, and any

*Supported by the Austrian Science Fund (FWF) Project F5506-N26.

${ }^{\dagger}$ Supported by the Austrian Science Fund (FWF) Project F5509-N26. 
efficient algorithm for dealing with functions of only very few variables becomes also efficient for functions of $s$ variables.

The main result of this paper is the observation that the $\varepsilon$-truncation dimension is surprisingly small for modest error demand $\varepsilon$ and the weights decaying sufficiently fast. For instance, for product weights

$$
\gamma_{\mathfrak{u}}=\prod_{j \in \mathfrak{u}} j^{-a}
$$

and the parameters $p=q=2$, we have the following upper bounds $k(\varepsilon)$ on $\operatorname{dim}^{\operatorname{trnc}}(\varepsilon)$ for $a=3,4,5$ :

\begin{tabular}{c||c|c|c|c|c|c||c}
$\varepsilon$ & $10^{-1}$ & $10^{-2}$ & $10^{-3}$ & $10^{-4}$ & $10^{-5}$ & $10^{-6}$ & \\
\hline$k(\varepsilon)$ & 2 & 5 & 12 & 31 & 79 & 198 & $a=3$ \\
\hline$k(\varepsilon)$ & 2 & 3 & 6 & 11 & 22 & 42 & $a=4$ \\
\hline$k(\varepsilon)$ & 1 & 2 & 4 & 6 & 11 & 18 & $a=5$
\end{tabular}

We stress that our definition of truncation dimension is different from the one proposed in statistical literature. There the dimension depends on a particular function via its ANOVA decomposition which, in general, cannot be computed. Moreover, for functions from spaces with ANOVA decomposition, small truncation dimension cannot be utilized unless the weights $\gamma_{\mathfrak{u}}$ are such that the anchored and ANOVA norms are equivalent. Such equivalence has recently been studied in [1, 2, 3, 4, 6] and, in particular, there is an equivalence independent of $s$ for product weights that are summable, with the equivalence constant bounded from above by

$$
\sum_{\mathfrak{u}} \gamma_{\mathfrak{u}}<\infty
$$

For product weights mentioned above we have

$$
\sum_{\mathfrak{u}} \gamma_{\mathfrak{u}}=\prod_{j=1}^{\infty}\left(1+j^{-a}\right)<\infty
$$

and hence the corresponding efficient algorithms for anchored spaces can also be used efficiently for ANOVA spaces.

The paper is structured as follows. In Section 2.1, we define the anchored spaces $F_{s, p, \gamma}$, and in Section 2.2 we outline the problem setting. We give our results for anchored spaces in Section 3, and discuss examples of special kinds of weights in Sections 3.1 and 3.2 .

\section{Basic Concepts}

\subsection{Anchored Spaces}

In this section, we briefly recall definitions and basic properties of $\gamma$-weighted anchored Sobolev spaces of $s$-variate functions. More detailed information can be found in [3, 4, 10,

Here we follow [10, Section 2]: For $p \in[1, \infty]$ let $F=W_{p, 0}^{1}([0,1])$ be the space of functions defined on $[0,1]$ that vanish at zero, are absolutely continuous, and have bounded derivative in the $L_{p}$ norm. We endow $F$ with the norm $\|f\|_{F}=\left\|f^{\prime}\right\|_{L_{p}}$ for $f \in F$.

For $s \in \mathbb{N}$ and

$$
[s]:=\{1,2, \ldots, s\},
$$


we will use $\mathfrak{u}, \mathfrak{v}$ to denote subsets of $[s]$, i.e.,

$$
\mathfrak{u}, \mathfrak{v} \subseteq[s]
$$

Moreover, for $\boldsymbol{x}=\left(x_{1}, x_{2}, \ldots, x_{s}\right) \in[0,1]^{s}$ and $\mathfrak{u} \subseteq[s],\left[\boldsymbol{x}_{\mathfrak{u}} ; \mathbf{0}_{-\mathfrak{u}}\right]$ denotes the $s$-dimensional vector with all $x_{j}$ for $j \notin \mathfrak{u}$ replaced by zero, i.e.,

$$
\left[\boldsymbol{x}_{\mathfrak{u}} ; \mathbf{0}_{-\mathfrak{u}}\right]=\left(y_{1}, y_{2}, \ldots, y_{s}\right) \quad \text { with } \quad y_{j}= \begin{cases}x_{j} & \text { if } j \in \mathfrak{u} \\ 0 & \text { if } j \notin \mathfrak{u}\end{cases}
$$

We also write $\boldsymbol{x}_{\mathfrak{u}}$ to denote the $|\mathfrak{u}|$-dimensional vector $\left(x_{j}\right)_{j \in \mathfrak{u}}$ and

$$
f^{(\mathfrak{u})}=\frac{\partial^{|\mathfrak{u}|} f}{\partial \boldsymbol{x}_{\mathfrak{u}}}=\prod_{j \in \mathfrak{u}} \frac{\partial}{\partial x_{j}} f \quad \text { with } \quad f^{(\emptyset)}=f .
$$

For $s \in \mathbb{N}$ and nonempty $\mathfrak{u} \subseteq[s]$ let $F_{\mathfrak{u}}$ be the completion of the space spanned by $f(\boldsymbol{x})=\prod_{j \in \mathfrak{u}} f_{j}\left(x_{j}\right)$ for $f_{j} \in F$ and $\boldsymbol{x}=\left(x_{1}, \ldots, x_{s}\right) \in[0,1]^{s}$, with the norm

$$
\|f\|_{F_{\mathfrak{u}}}=\left\|f^{(\mathfrak{u})}\right\|_{L_{p}}
$$

Note that $F_{\mathfrak{u}}$ is a space of functions with domain $[0,1]^{s}$ that depend only on the variables listed in $\mathfrak{u}$. Moreover, for any $f \in F_{\mathfrak{u}}$ and $\boldsymbol{x}=\left(x_{1}, \ldots, x_{s}\right), f(\boldsymbol{x})=0$ if $x_{j}=0$ for some $j \in \mathfrak{u}$. For $\mathfrak{u}=\emptyset$, let $F_{\mathfrak{u}}$ be the space of constant functions with the natural norm.

Consider next a sequence $\gamma=\left(\gamma_{\mathfrak{u}}\right)_{\mathfrak{u} \subseteq[s]}$ of non-negative real numbers, called weights. Since some weights could be zero, we will use

$$
\mathfrak{U}=\left\{\mathfrak{u} \subseteq[s]: \gamma_{\mathfrak{u}}>0\right\}
$$

to denote the collection of positive weights. For $p \in[1, \infty]$, we define the corresponding weighted anchored space

$$
F_{s, p, \gamma}=\operatorname{span}\left(\bigcup_{\mathfrak{u} \in \mathfrak{U}} F_{\mathfrak{u}}\right)
$$

with the norm

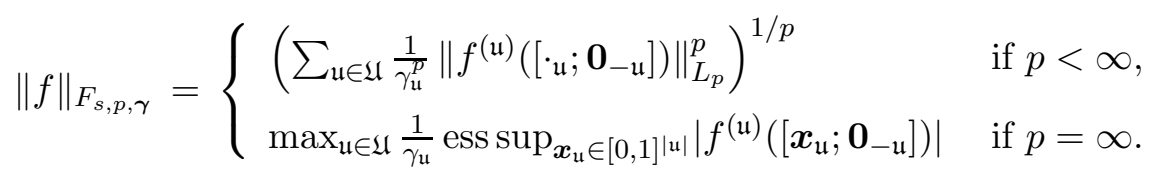

Remark 1 Some of the results of this paper can be extended to spaces of functions with countably many variables. In such cases, $[s]=\mathbb{N}$, the sets $\mathfrak{u}$ are finite subsets of $\mathbb{N}$, and $\boldsymbol{x}=\left(x_{j}\right)_{j \in \mathbb{N}}$ with $x_{j} \in[0,1]$. Moreover, the anchored space is the completion of $\operatorname{span}\left(\bigcup_{\mathfrak{u}} F_{\mathfrak{u}}\right)$ with respect to the norm given above.

An important class of weights is provided by product weights

$$
\gamma_{\mathfrak{u}}=\prod_{j \in \mathfrak{u}} \gamma_{j}
$$


for positive reals $\gamma_{j}$. When dealing with them, we will assume without any loss of generality that

$$
\gamma_{j} \geq \gamma_{j+1}>0 \text { for all } j .
$$

Note that for product weights we have $\mathfrak{U}=2^{[s]}=\{\mathfrak{u}: \mathfrak{u} \subseteq[s]\}$.

For $p=2, F_{s, 2, \gamma}$ is a reproducing kernel Hilbert space with kernel

$$
K(\boldsymbol{x}, \boldsymbol{y})=\sum_{\mathfrak{u} \in \mathfrak{U}} \gamma_{\mathfrak{u}}^{2} \prod_{j \in \mathfrak{u}} \min \left(x_{j}, y_{j}\right)
$$

for $\boldsymbol{x}=\left(x_{1}, \ldots, x_{s}\right)$ and analogously for $\boldsymbol{y}$, which for product weights reduces to

$$
K(\boldsymbol{x}, \boldsymbol{y})=\prod_{j=1}^{s}\left(1+\gamma_{j}^{2} \min \left(x_{j}, y_{j}\right)\right)
$$

\subsection{The Function Approximation Problem}

We follow [10]. Let $q \in[1, \infty]$. For $\mathfrak{u} \in \mathfrak{U}$, let $S_{\mathfrak{u}}: F_{\mathfrak{u}} \rightarrow L_{q}\left([0,1]^{|\mathfrak{u}|}\right)$ be the embedding operator,

$$
S_{\mathfrak{u}}\left(f_{\mathfrak{u}}\right)=f_{\mathfrak{u}} \quad \text { for all } f_{\mathfrak{u}} \in F_{\mathfrak{u}} .
$$

It is well known that

$$
\left\|S_{\mathfrak{u}}\right\|=\|S\|^{|\mathfrak{u}|}
$$

for the space $F_{\mathfrak{u}}$, where $S: F \rightarrow L_{q}([0,1])$ is the univariate embedding operator.

Let further $\mathcal{L}_{q}$ be a normed linear space such that $F_{s, p, \gamma}$ is its subspace and the norm $\|\cdot\|_{\mathcal{L}_{q}}$ is such that

$$
\left\|f_{\mathfrak{u}}\right\|_{\mathcal{L}_{q}}=\left\|f_{\mathfrak{u}}\right\|_{L_{q}\left([0,1]^{|\mathfrak{u}|}\right)} \quad \text { for all } f_{\mathfrak{u}} \in F_{\mathfrak{u}} .
$$

Denote by $S_{s}$ the embedding operator

$$
S_{s}: F_{s, p, \gamma} \rightarrow \mathcal{L}_{q}, \quad S_{s}(f)=f .
$$

In order to make sure that $S_{s}$ is continuous, we assume from now on that

$$
\sum_{\mathfrak{u} \in \mathfrak{U}}\left(\left(\frac{q}{p^{*}}+1\right)^{-|\mathfrak{u}| / q} \gamma_{\mathfrak{u}}\right)^{p^{*}}<\infty
$$

where here and throughout this paper $p^{*}$ denotes the conjugate of $p$, i.e., $1 / p+1 / p^{*}=1$. To see that this condition indeed ensures continuity of $S_{s}$, we recall the following proposition from [10].

Proposition 1 We have

$$
\|S\| \leq\left(\frac{q}{p^{*}}+1\right)^{-1 / q} \quad \text { and } \quad\left\|S_{s}\right\| \leq\left[\sum_{\mathfrak{u} \in \mathfrak{U}}\left(\left(\frac{q}{p^{*}}+1\right)^{-|\mathfrak{u}| / q} \gamma_{\mathfrak{u}}\right)^{p^{*}}\right]^{1 / p^{*}} .
$$


Note that for product weights we have

$$
\sum_{\mathfrak{u} \in \mathfrak{U}}\left(\left(\frac{q}{p^{*}}+1\right)^{-|\mathfrak{u}| / q} \gamma_{\mathfrak{u}}\right)^{p^{*}}=\prod_{j=1}^{s}\left(1+\gamma_{j}^{p^{*}}\left(\frac{q}{p^{*}}+1\right)^{-p^{*} / q}\right) .
$$

We are interested in algorithms for approximating $f \in F_{s, p, \boldsymbol{\gamma}}$. In this paper we only consider algorithms that use so-called standard information, i.e., which only use function evaluations as the allowed information class, and which have the form

$$
A_{s}(f)=\phi\left(f\left(\boldsymbol{x}_{1}\right), \ldots, f\left(\boldsymbol{x}_{n}\right)\right)
$$

for $\boldsymbol{x}_{j} \in[0,1]^{s}$, where $\phi: \mathbb{R}^{n} \rightarrow \mathcal{L}_{q}$. An important class of algorithms is provided by the class of linear algorithms which are of the form

$$
A_{s}(f)=\sum_{j=1}^{n} f\left(\boldsymbol{x}_{j}\right) g_{j}
$$

for $g_{j} \in F_{s, p, \boldsymbol{\gamma}}$. We study the worst case setting, where the error of an algorithm $A_{s}$ is the operator norm of $S_{s}-A_{s}$, i.e.,

$$
e\left(A_{s} ; F_{s, p, \gamma}\right):=\left\|S_{s}-A_{s}\right\|=\sup _{\|f\|_{F_{s, p, \gamma} \leq 1}}\left\|f-A_{s}(f)\right\|_{\mathcal{L}_{q}} .
$$

\section{Anchored Decomposition and Truncation Dimen- sion}

It is well known, see, e.g., [8], that any $f \in F_{s, p, \gamma}$ has the unique anchored decomposition

$$
f=\sum_{\mathfrak{u} \in \mathfrak{U}} f_{\mathfrak{u}}
$$

where $f_{\mathfrak{u}}$ is an element of $F_{\mathfrak{u}}$, depends only on $x_{j}$ for $j \in \mathfrak{u}$, and

$$
f_{\mathfrak{u}}(\boldsymbol{x})=0 \quad \text { if } x_{j}=0 \text { for some } j \in \mathfrak{u} .
$$

For the empty set $\mathfrak{u}, f_{\emptyset}$ is a constant function. We stress that in general we do not know what the elements $f_{\mathfrak{u}}$ are and algorithms are only allowed to evaluate the original function $f$.

The anchored decomposition has the following important properties, see, e.g., [3]:

$$
\begin{gathered}
f^{(\mathfrak{u})}\left(\left[\cdot \cdot_{\mathfrak{u}} ; \mathbf{0}_{-\mathfrak{u}}\right]\right) \equiv f_{\mathfrak{u}}^{(\mathfrak{u})} \\
f_{\mathfrak{u}} \equiv 0 \quad \text { iff } \quad f^{(\mathfrak{u})}\left(\left[\cdot \mathfrak{u} ; \mathbf{0}_{-\mathfrak{u}}\right]\right) \equiv 0 \\
\|f\|_{F_{s, p, \gamma}}=\left\|\sum_{\mathfrak{u} \in \mathfrak{U}} f_{\mathfrak{u}}\right\|_{F_{s, p, \gamma}}=\left(\sum_{\mathfrak{u} \in \mathfrak{U}} \gamma_{\mathfrak{u}}^{-p}\left\|f_{\mathfrak{u}}^{(\mathfrak{u})}\right\|_{L_{p}}^{p}\right)^{1 / p} \quad \text { for } p<\infty,
\end{gathered}
$$

and

$$
\|f\|_{F_{s, \infty, \gamma}}=\max _{\mathfrak{u} \in \mathfrak{U}} \frac{\left\|f_{\mathfrak{u}}^{(\mathfrak{u})}\right\|_{L_{\infty}}}{\gamma_{\mathfrak{u}}} \quad \text { for } p=\infty
$$




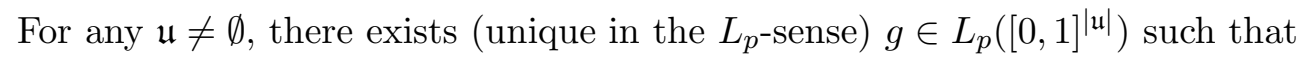

$$
f_{\mathfrak{u}}(\boldsymbol{x})=\int_{[0,1]|\mathfrak{u}|} g(\boldsymbol{t}) \prod_{j \in \mathfrak{u}} 1_{\left[0, x_{j}\right)}\left(t_{j}\right) \mathrm{d} \boldsymbol{t} \quad \text { and } \quad f_{\mathfrak{u}}^{(\mathfrak{u})}=g
$$

where $1_{J}(t)$ is the characteristic function of the set $J$, i.e., $1_{J}(t)=1$ if $t \in J$ and 0 otherwise.

Moreover, for any $\mathfrak{u}$,

$$
f\left(\left[\cdot_{\mathfrak{u}} ; \mathbf{0}_{-\mathfrak{u}}\right]\right)=\sum_{\mathfrak{v} \subseteq \mathfrak{u}} f_{\mathfrak{v}}
$$

In particular, for $k<s$ we have

$$
f\left(\left[\boldsymbol{x}_{[k]} ; \mathbf{0}_{-[k]}\right]\right)=f\left(x_{1}, \ldots, x_{k}, 0, \ldots, 0\right)=\sum_{\mathfrak{v} \subseteq[k]} f_{\mathfrak{v}}(\boldsymbol{x})
$$

which allows us to compute samples and approximate the truncated function

$$
f_{k}\left(x_{1}, \ldots, x_{k}\right):=\sum_{\mathfrak{v} \subseteq[k]} f_{\mathfrak{v}}(\boldsymbol{x}) .
$$

Moreover, $f_{k} \in F_{k, p, \gamma} \subseteq F_{s, p, \gamma}$ and

$$
\left\|f_{k}\right\|_{F_{k, p, \gamma}}=\left\|f\left(\left[\cdot[k] ; \mathbf{0}_{-[k]}\right]\right)\right\|_{F_{s, p, \gamma}}=\left\|\sum_{\mathfrak{u} \subseteq[k]} f_{\mathfrak{u}}\right\|_{F_{s, p, \gamma}} .
$$

This leads to the following concept.

Definition 1 For a given error demand $\varepsilon>0$, by $\varepsilon$-truncation dimension for the approximation problem (or truncation dimension for short), denoted by $\operatorname{dim}^{\text {trnc }}(\varepsilon)$, we mean the smallest integer $k$ such that

$$
\left\|\sum_{\mathfrak{u} \nsubseteq[k]} f_{\mathfrak{u}}\right\|_{\mathcal{L}_{q}} \leq \varepsilon\left\|\sum_{\mathfrak{u} \mathbb{\complement}[k]} f_{\mathfrak{u}}\right\|_{F_{s, p, \gamma}} \quad \text { for all } f=\sum_{\mathfrak{u} \in \mathfrak{U}} f_{\mathfrak{u}} \in F_{s, p, \boldsymbol{\gamma}} .
$$

We have the following upper bound on the truncation dimension.

Theorem 1 We have

$$
\operatorname{dim}^{\operatorname{trnc}}(\varepsilon) \leq \min \left\{k:\left(\sum_{\mathfrak{u} \notin[k]} \frac{\gamma_{\mathfrak{u}}^{p^{*}}}{\left(\frac{q}{p^{*}}+1\right)^{p^{*}|\mathfrak{u}| / q}}\right)^{1 / p^{*}} \leq \varepsilon\right\} \quad \text { for } p>1,
$$

and

$$
\operatorname{dim}^{\operatorname{trnc}}(\varepsilon)=\min \left\{k: \max _{\mathfrak{u} \mathbb{E}[k]} \gamma_{\mathfrak{u}} \leq \varepsilon\right\} \quad \text { for } p=1 .
$$

Here $\sum_{\mathfrak{u} \nsubseteq[k]}$ means summation over all $\mathfrak{u} \subseteq[s]$ with $\mathfrak{u} \nsubseteq[k]$ and similarly for $\max _{\mathfrak{u} \nsubseteq[k]}$. 
The proof of the theorem is in the proof of the next, Theorem 2.

For given $k<s$, let $A_{k}$ be an algorithm for approximating functions from the space $F_{k, p, \gamma}$. We use it to define the following approximation algorithms for the original space $F_{s, p, \gamma}$,

$$
A_{s, k}^{\mathrm{trnc}}(f)=A_{k}\left(f\left(\left[\cdot[k] ; \mathbf{0}_{-[k]}\right]\right)\right)=A_{k}\left(f_{k}\right) .
$$

Clearly, the algorithms $A_{s, k}^{\text {trnc }}$ are well defined.

We have the following result.

Theorem 2 For every $k<s$, the worst case error of $A_{s, k}^{\mathrm{trnc}}$ is bounded by

$$
e\left(A_{s, k}^{\mathrm{trnc}} ; F_{s, p, \gamma}\right) \leq\left(\left[e\left(A_{k} ; F_{k, p, \gamma}\right)\right]^{p^{*}}+\sum_{\mathfrak{u} \notin[k]} \frac{\gamma_{\mathfrak{u}}^{p^{*}}}{\left(\frac{q}{p^{*}}+1\right)^{p^{*}|\mathfrak{u}| / q}}\right)^{1 / p^{*}} \text { for } p>1
$$

and by

$$
e\left(A_{s, k}^{\mathrm{trnc}} ; F_{s, 1, \gamma}\right) \leq \max \left(e\left(A_{k} ; F_{k, 1, \gamma}\right), \max _{\mathfrak{u} \subset[k]} \gamma_{\mathfrak{u}}\right) \quad \text { for } p=1 .
$$

Moreover, if $k \geq \operatorname{dim}^{\operatorname{trnc}}(\varepsilon)$ then

$$
e\left(A_{s, k}^{\mathrm{trnc}} ; F_{s, p, \gamma}\right) \leq\left(\left[e\left(A_{k} ; F_{k, p, \gamma}\right)\right]^{p^{*}}+\varepsilon^{p^{*}}\right)^{1 / p^{*}} .
$$

Proof. We prove the theorem for $p>1$ only since the proof for $p=1$ is very similar. Let us first assume that $1<p<\infty$. For any $f \in F_{s, p, \gamma}$ it holds that

$$
\begin{aligned}
\left\|S_{s}(f)-A_{s, k}^{\operatorname{trnc}}(f)\right\|_{\mathcal{L}_{q}} & =\left\|S_{s}(f)-A_{k}\left(f_{k}\right)\right\|_{\mathcal{L}_{q}} \\
& =\left\|S_{k}\left(f_{k}\right)-A_{k}\left(f_{k}\right)+\sum_{\mathfrak{u} \nsubseteq[k]} S_{\mathfrak{u}}\left(f_{\mathfrak{u}}\right)\right\|_{\mathcal{L}_{q}} \\
& \leq e\left(A_{k} ; F_{k, p, \gamma}\right)\left\|f_{k}\right\|_{F_{k, p, \gamma}}+\sum_{\mathfrak{u} \nsubseteq[k]}\left\|S_{\mathfrak{u}}\left(f_{\mathfrak{u}}\right)\right\|_{\mathcal{L}_{q}} \\
& \leq e\left(A_{k} ; F_{k, p, \gamma}\right)\left\|f_{k}\right\|_{F_{k, p, \gamma}}+\sum_{\mathfrak{u} \mathbb{E}[k]}\left\|f_{\mathfrak{u}}\right\|_{F_{\mathfrak{u}}}\left\|S_{\mathfrak{u}}\right\| .
\end{aligned}
$$

We now have

$$
\begin{aligned}
& \sum_{\mathfrak{u} \nsubseteq[k]}\left\|f_{\mathfrak{u}}\right\|_{F_{\mathfrak{u}}}\left\|S_{\mathfrak{u}}\right\|=\sum_{\mathfrak{u} \nsubseteq[k]} \gamma_{\mathfrak{u}}^{-1}\left\|f_{\mathfrak{u}}\right\|_{F_{\mathfrak{u}}} \gamma_{\mathfrak{u}}\left\|S_{\mathfrak{u}}\right\| \\
& \leq\left[\sum_{\mathfrak{u} \nsubseteq[k]}\left(\frac{\left\|f_{\mathfrak{u}}\right\|_{F_{\mathfrak{u}}}}{\gamma_{\mathfrak{u}}}\right)^{p}\right]^{1 / p}\left[\sum_{\mathfrak{u} \nsubseteq[k]}\left(\gamma_{\mathfrak{u}}\left\|S_{\mathfrak{u}}\right\|\right)^{p^{*}}\right]^{1 / p^{*}} \\
& \leq\left[\sum_{\mathfrak{u} \nsubseteq[k]}\left(\frac{\left\|f_{\mathfrak{u}}\right\|_{F_{\mathfrak{u}}}}{\gamma_{\mathfrak{u}}}\right)^{p}\right]^{1 / p}\left[\sum_{\mathfrak{u} \nsubseteq[k]}\left(\gamma_{\mathfrak{u}}\left(\frac{q}{p^{*}}+1\right)^{-|\mathfrak{u}| / q}\right)^{p^{*}}\right]^{1 / p^{*}}
\end{aligned}
$$

where we used Proposition 1 in the last step. 
Hence, putting together, we get

$$
\begin{aligned}
\left\|S_{s}(f)-A_{s, k}^{\operatorname{trnc}}(f)\right\|_{\mathcal{L}_{q}} \leq & e\left(A_{k} ; F_{k, p, \gamma}\right)\left(\sum_{\mathfrak{u} \subseteq[k]} \gamma_{\mathfrak{u}}^{-p}\left\|f_{\mathfrak{u}}^{(\mathfrak{u})}\right\|_{L_{p}}^{p}\right)^{1 / p} \\
& +\left(\sum_{\mathfrak{u} \mathbb{\complement}[k]} \frac{\gamma_{\mathfrak{u}}^{p^{*}}}{\left(\frac{q}{p^{*}}+1\right)^{p^{*}|\mathfrak{u}| / q}}\right)^{1 / p^{*}}\left(\sum_{\mathfrak{u} \mathbb{\complement}[k]} \gamma_{\mathfrak{u}}^{-p}\left\|f_{\mathfrak{u}}^{(\mathfrak{u})}\right\|_{L_{p}}^{p}\right)^{1 / p} .
\end{aligned}
$$

Using the Hölder inequality once more, we obtain

$$
\begin{aligned}
& \left\|S_{s}(f)-A_{s, k}^{\operatorname{trnc}}(f)\right\|_{\mathcal{L}_{q}} \\
& \leq\left(\sum_{\mathfrak{u} \subseteq[s]} \gamma_{\mathfrak{u}}^{-p}\left\|f_{\mathfrak{u}}^{(\mathfrak{u})}\right\|_{L_{p}}^{p}\right)^{1 / p}\left(\left[e\left(A_{k} ; F_{k, p, \gamma}\right)\right]^{p^{*}}+\sum_{\mathfrak{u} \subseteq[k]} \frac{\gamma_{\mathfrak{u}}^{p^{*}}}{\left(\frac{q}{p^{*}}+1\right)^{p^{*}|\mathfrak{u}| / q}}\right)^{1 / p^{*}} \\
& =\|f\|_{F_{s, p, \gamma}}\left(\left[e\left(A_{k} ; F_{k, p, \gamma}\right)\right]^{p^{*}}+\sum_{\mathfrak{u} \nsubseteq[k]} \frac{\gamma_{\mathfrak{u}}^{p^{*}}}{\left(\frac{q}{p^{*}}+1\right)^{p^{*}|\mathfrak{u}| / q}}\right)^{1 / p^{*}} .
\end{aligned}
$$

This shows the result for $1<p<\infty$. For $p=\infty$, the result is obtained by letting $p \rightarrow \infty$. This completes the proof.

We now apply this theorem to two important classes of weights: product weights and product order-dependent weights.

\subsection{Product Weights}

We assume in this section that the weights have the following product form

$$
\gamma_{\mathfrak{u}}=\prod_{j \in \mathfrak{u}} \gamma_{j} \quad \text { for } \quad 1 \geq \gamma_{j} \geq \gamma_{j+1}>0
$$

introduced in [9]. Here the empty product is considered to be 1, i.e., $\gamma_{\emptyset}=1$. As already mentioned, for product weights we always have $\mathfrak{U}=2^{[s]}$.

Proposition 2 For product weights and $k<s$, the truncation error is bounded by

$$
\begin{aligned}
\left(\sum_{\mathfrak{u} \notin[k]} \frac{\gamma_{\mathfrak{u}}^{p^{*}}}{\left(\frac{q}{p^{*}}+1\right)^{p^{*}|\mathfrak{u}| / q}}\right)^{1 / p^{*}} \leq & \prod_{j=1}^{s}\left(1+\frac{\gamma_{j}^{p^{*}}}{\left(\frac{q}{p^{*}}+1\right)^{p^{*} / q}}\right)^{1 / p^{*}} \\
& \times\left(1-\exp \left(\frac{-1}{\left(\frac{q}{p^{*}}+1\right)^{p^{*} / q}} \sum_{j=k+1}^{s} \gamma_{j}^{p^{*}}\right)\right)^{1 / p^{*}}
\end{aligned}
$$

for $p>1$, and it is equal to

$$
\max _{\mathfrak{u} \nsubseteq[k]} \gamma_{\mathfrak{u}} \quad \text { for } p=1
$$


Proof. The proof for $p=1$ is trivial. For $p>1$, we have

$$
\begin{aligned}
& \sum_{\mathfrak{u} \subseteq[k]} \frac{\gamma_{\mathfrak{u}}^{p^{*}}}{\left(\frac{q}{p^{*}}+1\right)^{p^{*}|\mathfrak{u}| / q}}=\sum_{\mathfrak{u} \subseteq[s]} \frac{\gamma_{\mathfrak{u}}^{p^{*}}}{\left(\frac{q}{p^{*}}+1\right)^{p^{*}|\mathfrak{u}| / q}}-\sum_{\mathfrak{u} \subseteq[k]} \frac{\gamma_{\mathfrak{u}}^{p^{*}}}{\left(\frac{q}{p^{*}}+1\right)^{p^{*}|\mathfrak{u}| / q}} \\
& \quad=\prod_{j=1}^{s}\left(1+\frac{\gamma_{j}^{p^{*}}}{\left(\frac{q}{p^{*}}+1\right)^{p^{*} / q}}\right)-\prod_{j=1}^{k}\left(1+\frac{\gamma_{j}^{p^{*}}}{\left(\frac{q}{p^{*}}+1\right)^{p^{*} / q}}\right) \\
& \quad=\prod_{j=1}^{s}\left(1+\frac{\gamma_{j}^{p^{*}}}{\left(\frac{q}{p^{*}}+1\right)^{p^{*} / q}}\right)\left(1-\prod_{j=k+1}^{s}\left(1+\frac{\gamma_{j}^{p^{*}}}{\left(\frac{q}{p^{*}}+1\right)^{p^{*} / q}}\right)^{-1}\right) .
\end{aligned}
$$

We have

$$
\begin{aligned}
1-\prod_{j=k+1}^{s}\left(1+\frac{\gamma_{j}^{p^{*}}}{\left(\frac{q}{p^{*}}+1\right)^{p^{*} / q}}\right)^{-1} & =1-\exp \left(-\sum_{j=k+1}^{s} \log \left(1+\frac{\gamma_{j}^{p^{*}}}{\left(\frac{q}{p^{*}}+1\right)^{p^{*} / q}}\right)\right) \\
& \leq 1-\exp \left(-\sum_{j=k+1}^{s} \frac{\gamma_{j}^{p^{*}}}{\left(\frac{q}{p^{*}}+1\right)^{p^{*} / q}}\right),
\end{aligned}
$$

where $\log$ denotes the natural logarithm, and the last inequality is due to $\log (1+x) \leq x$ for all $x>-1$. This completes the proof.

We have the following corollaries:

Corollary 1 Consider product weights. Then $\operatorname{dim}^{\text {trnc }}(\varepsilon)$ is bounded from above by

$$
\min \left\{k: 1-\exp \left(\frac{-1}{\left(\frac{q}{p^{*}}+1\right)^{p^{*} / q}} \sum_{j=k+1}^{s} \gamma_{j}^{p^{*}}\right) \leq \frac{\varepsilon^{p^{*}}}{\prod_{j=1}^{s}\left(1+\frac{\gamma_{j}^{p^{*}}}{\left(\frac{q}{p^{*}}+1\right)^{p^{*} / q}}\right)}\right\}
$$

for $p>1$, and is equal to

$$
\min \left\{k: \max _{\mathfrak{u} \nsubseteq[s]} \gamma_{\mathfrak{u}} \leq \varepsilon\right\}
$$

for $p=1$.

Corollary 2 Consider product weights and $k<s$.

Then the error $e\left(A_{s, k}^{\mathrm{trnc}} ; F_{s, p, \gamma}\right)$ is bounded from above by

$$
\begin{aligned}
& \left(\left[e\left(A_{k} ; F_{k, p, \gamma}\right)\right]^{p^{*}}\right. \\
& \left.+\prod_{j=1}^{s}\left(1+\frac{\gamma_{j}^{p^{*}}}{\left(\frac{q}{p^{*}}+1\right)^{p^{*} / q}}\right)\left(1-\exp \left(\frac{-1}{\left(\frac{q}{p^{*}}+1\right)^{p^{*} / q}} \sum_{j=k+1}^{s} \gamma_{j}^{p^{*}}\right)\right)\right)^{1 / p^{*}}
\end{aligned}
$$


for $p>1$, and by

$$
\max \left(e\left(A_{k} ; F_{k, 1, \gamma}\right), \max _{\mathfrak{u} \nsubseteq[k]} \gamma_{\mathfrak{u}}\right)
$$

for $p=1$. Note that if $\gamma_{j} \leq 1$ for all $j$ then $\max _{\mathfrak{u} \nsubseteq[s]} \gamma_{\mathfrak{u}}=\gamma_{k+1}$.

Therefore, for the worst case error of $A_{s, k}^{\text {trnc }}$ not to exceed the error demand $\varepsilon>0$, it is enough to choose $k=k(\varepsilon)$ so that

$$
1-\exp \left(\frac{-1}{\left(\frac{q}{p^{*}}+1\right)^{p^{*} / q}} \sum_{j=k+1}^{s} \gamma_{j}^{p^{*}}\right) \leq \frac{1}{2} \varepsilon^{p^{*}} \prod_{j=1}^{s}\left(1+\frac{\gamma_{j}^{p^{*}}}{\left(\frac{q}{p^{*}}+1\right)^{p^{*} / q}}\right)^{-1}
$$

(or $\gamma_{k+1} \leq \varepsilon$ for $p=1$ ), and next to choose $n=n(\varepsilon)$ so that

$$
e\left(A_{k} ; F_{k, p, \gamma}\right) \leq \frac{\varepsilon}{2^{1 / p^{*}}} .
$$

Clearly the inequality (6) for $p>1$ is equivalent to

$$
\sum_{j=k+1}^{s} \gamma_{j}^{p^{*}} \leq-\left(\frac{q}{p^{*}}+1\right)^{p^{*} / q} \log \left(1-\frac{1}{2} \varepsilon^{p^{*}} \prod_{j=1}^{s}\left(1+\frac{\gamma_{j}^{p^{*}}}{\left(\frac{q}{p^{*}}+1\right)^{p^{*} / q}}\right)^{-1}\right) .
$$

Example 1 Consider large $s$ including $s=\infty$ and

$$
\gamma_{\mathfrak{u}}=\prod_{j \in \mathfrak{u}} j^{-a} \text { for } a>1 / p^{*} .
$$

For $p=1$, we have

$$
\operatorname{dim}^{\operatorname{trnc}}(\varepsilon)=\left\lceil\varepsilon^{-1 / a}-1\right\rceil
$$

In particular we have

\begin{tabular}{c||c|c|c|c|c|c||c}
$\varepsilon$ & $10^{-1}$ & $10^{-2}$ & $10^{-3}$ & $10^{-4}$ & $10^{-5}$ & $10^{-6}$ & \\
\hline $\operatorname{dim}^{\operatorname{trnc}}(\varepsilon)$ & 3 & 9 & 31 & 99 & 316 & 999 & $a=2$ \\
\hline $\operatorname{dim}^{\operatorname{trnc}}(\varepsilon)$ & 2 & 4 & 9 & 21 & 46 & 99 & $a=3$ \\
\hline $\operatorname{dim}^{\operatorname{trnc}}(\varepsilon)$ & 1 & 3 & 5 & 9 & 17 & 31 & $a=4$ \\
\hline $\operatorname{dim}^{\operatorname{trnc}}(\varepsilon)$ & 1 & 2 & 3 & 6 & 9 & 15 & $a=5$
\end{tabular}

Consider next $p>1$. Unlike in the case $p=1$, we do not know the exact values of the truncation dimension. However, we have its upper bounds $k(\varepsilon)$ that are small,

$$
\operatorname{dim}^{\text {trnc }}(\varepsilon) \leq k(\varepsilon) .
$$

We use the estimate

$$
\frac{(k+1)^{-a p^{*}+1}}{a p^{*}-1}=\int_{k+1}^{\infty} x^{-a p^{*}} \mathrm{~d} x<\sum_{j=k+1}^{\infty} j^{-a p^{*}} \leq \int_{k+1 / 2}^{\infty} x^{-a p^{*}} \mathrm{~d} x=\frac{(k+1 / 2)^{-a p^{*}+1}}{a p^{*}-1} .
$$

Note that the relative error when using the upper bound to approximate the sum is bounded by

$$
\frac{a p^{*}-1}{2 k+1}+O\left(k^{-2}\right)
$$


and is small for large $k$. To satisfy (7), it is enough to take $k=k(\varepsilon)$ given by

$$
k=\left\lceil\left(\frac{-\left(\frac{q}{p^{*}}+1\right)^{-p^{*} / q}\left(a p^{*}-1\right)^{-1}}{\log \left(1-\frac{\varepsilon^{*}}{2} \prod_{j=1}^{s}\left(1+\frac{j^{-a p^{*}}}{\left(\frac{q}{p^{*}}+1\right)^{p^{*} / q}}\right)^{-1}\right)}\right)^{1 /\left(a p^{*}-1\right)}-\frac{1}{2}\right]
$$

For $p=p^{*}=2$, which corresponds to the classical Hilbert space setting, we have

$$
k(\varepsilon)=\left\lceil\left(\frac{-\left(\frac{q}{2}+1\right)^{-2 / q}(2 a-1)^{-1}}{\log \left(1-\frac{\varepsilon^{2}}{2} \prod_{j=1}^{s}\left(1+\frac{j^{-2 a}}{\left(\frac{q}{2}+1\right)^{2 / q}}\right)^{-1}\right)}\right)^{1 /(2 a-1)}-\frac{1}{2}\right\rceil .
$$

If also $q=2$, then

$$
k(\varepsilon)=\left\lceil\left(-2(2 a-1) \log \left(1-\frac{\varepsilon^{2}}{2} \prod_{j=1}^{s}\left(1+\frac{j^{-2 a}}{2}\right)^{-1}\right)\right)^{-1 /(2 a-1)}-\frac{1}{2}\right\rceil .
$$

Since $s$ could be huge or $s=\infty$, in calculating the values of $k(\varepsilon)$, we slightly overestimated the product $\prod_{j=1}^{s}\left(1+\frac{j^{-2 a}}{2}\right)$ in the following way:

$$
\begin{aligned}
\prod_{j=1}^{s}\left(1+\frac{j^{-2 a}}{2}\right) & \leq \prod_{j=1}^{\infty}\left(1+\frac{j^{-2 a}}{2}\right) \leq \prod_{j=1}^{1000}\left(1+\frac{j^{-2 a}}{2}\right) \exp \left(\sum_{j=1001}^{\infty} \frac{j^{-2 a}}{2}\right) \\
& \leq \prod_{j=1}^{1000}\left(1+\frac{j^{-2 a}}{2}\right) \exp \left(\frac{1}{2} \int_{1000.5}^{\infty} x^{-2 a} \mathrm{~d} x\right) \\
& =\prod_{j=1}^{1000}\left(1+\frac{j^{-2 a}}{2}\right) \exp \left(\frac{1}{2(2 a-1)} 1000.5^{-2 a+1}\right) .
\end{aligned}
$$

This gave us the following estimations for $\prod_{j=1}^{s}\left(1+\frac{j^{-2 a}}{2}\right)$ for $p=2$ :

$$
1.56225 \text { for } a=2, \quad 1.51302 \text { for } a=3, \quad 1.50306 \text { for } a=4, \quad 1.50075 \text { for } a=5 \text {. }
$$

Below we give values of $k(\varepsilon)$ for $a=2,3,4,5$ and $p=q=2$ using the estimates above.

\begin{tabular}{c||c|c|c|c|c|c||c}
$\varepsilon$ & $10^{-1}$ & $10^{-2}$ & $10^{-3}$ & $10^{-4}$ & $10^{-5}$ & $10^{-6}$ & \\
\hline$k(\varepsilon)$ & 4 & 17 & 80 & 373 & 1733 & 8045 & $a=2$ \\
\hline$k(\varepsilon)$ & 2 & 5 & 12 & 31 & 79 & 198 & $a=3$ \\
\hline$k(\varepsilon)$ & 2 & 3 & 6 & 11 & 22 & 42 & $a=4$ \\
\hline$k(\varepsilon)$ & 1 & 2 & 4 & 6 & 11 & 18 & $a=5$
\end{tabular}


It is clear that $k(\varepsilon)$ decreases with increasing $a$. To check whether the estimates above are sharp, we also calculated $k(\varepsilon)$ for $s=1000000$ directly by computing

$$
\prod_{j=1}^{s}\left(1+\frac{j^{-2 a}}{2}\right)-\prod_{j=1}^{k}\left(1+\frac{j^{-2 a}}{2}\right)
$$

and choosing the smallest $k$ for which the difference above is not greater than $\varepsilon^{2} / 2$. The values of $k(\varepsilon)$ obtained this way are exactly the same.

We now consider $p=\infty$ and $q=2$. By computing

$$
\prod_{j=1}^{s}\left(1+\frac{j^{-a}}{\sqrt{3}}\right)-\prod_{j=1}^{k}\left(1+\frac{j^{-a}}{\sqrt{3}}\right)
$$

we obtained the following values of $k(\varepsilon)$ for $s=1000000$, which is the smallest $k$ for which the difference above is not greater than $\varepsilon / 2$.

\begin{tabular}{c||c|c|c|c|c|c||c}
$\varepsilon$ & $10^{-1}$ & $10^{-2}$ & $10^{-3}$ & $10^{-4}$ & $10^{-5}$ & $10^{-6}$ & \\
\hline$k(\varepsilon)$ & 3 & 10 & 32 & 101 & 319 & 1010 & $a=3$ \\
\hline$k(\varepsilon)$ & 2 & 4 & 9 & 19 & 40 & 86 & $a=4$ \\
\hline$k(\varepsilon)$ & 1 & 3 & 5 & 8 & 15 & 26 & $a=5$
\end{tabular}

We do not present the values of $k(\varepsilon)$ for $a=2$ since they are too large to be of practical interest. For instance $k\left(10^{-3}\right)=2069$ and $k\left(10^{-4}\right)=7230$.

For some particular values of $q$, the norm of the embedding operator $S$ is known, as for example for $q=1$, in which case it equals $\left(1+p^{*}\right)^{-1 / p^{*}}$.

Let us now consider the case $p=p^{*}=2, s=1000000$, and $q=1$. In this case we obtain from (8):

\begin{tabular}{c||c|c|c|c|c|c||c}
$\varepsilon$ & $10^{-1}$ & $10^{-2}$ & $10^{-3}$ & $10^{-4}$ & $10^{-5}$ & $10^{-6}$ & \\
\hline$k(\varepsilon)$ & 4 & 16 & 76 & 354 & 1643 & 7628 & $a=2$ \\
\hline$k(\varepsilon)$ & 2 & 5 & 12 & 30 & 76 & 192 & $a=3$ \\
\hline$k(\varepsilon)$ & 2 & 3 & 6 & 11 & 21 & 41 & $a=4$ \\
\hline$k(\varepsilon)$ & 1 & 2 & 4 & 6 & 10 & 17 & $a=5$
\end{tabular}

For comparison, we also consider the values of $k(\varepsilon)$, by using the precise formula for $\left\|S_{\mathfrak{u}}\right\|=$ $\|S\|^{|\mathfrak{u}|}=\left(1+p^{*}\right)^{-|\mathfrak{u}| / p^{*}}$ in the proof of Theorem [2, This yields, instead of (8) :

$$
k(\varepsilon)=\left\lceil\left(-3(2 a-1) \log \left(1-\frac{\varepsilon^{2}}{2} \prod_{j=1}^{s}\left(1+\frac{j^{-2 a}}{3}\right)^{-1}\right)\right)^{-1 /(2 a-1)}-\frac{1}{2}\right\rceil .
$$

Then we obtain from (9):

\begin{tabular}{c||c|c|c|c|c|c||c}
$\varepsilon$ & $10^{-1}$ & $10^{-2}$ & $10^{-3}$ & $10^{-4}$ & $10^{-5}$ & $10^{-6}$ & \\
\hline$k(\varepsilon)$ & 3 & 14 & 67 & 312 & 1449 & 6727 & $a=2$ \\
\hline$k(\varepsilon)$ & 2 & 4 & 11 & 28 & 71 & 178 & $a=3$ \\
\hline$k(\varepsilon)$ & 1 & 3 & 5 & 10 & 20 & 39 & $a=4$ \\
\hline$k(\varepsilon)$ & 1 & 2 & 4 & 6 & 10 & 17 & $a=5$
\end{tabular}

We see that the values of $k(\varepsilon)$ computed using the precise value of $\|S\|$ are lower than our general bounds, but not too much. 


\subsection{Product Order-Dependent Weights}

We assume in this section that the weights have the following product order-dependent (POD) form,

$$
\gamma_{\mathfrak{u}}=c_{1}(|\mathfrak{u}| !)^{b} \prod_{j \in \mathfrak{u}} \gamma_{j}
$$

introduced in [7]. Here $c_{1}$ is a positive constant. Since the truncation error for $p=1$ is $\max _{\mathfrak{u} \notin[k]} \gamma_{\mathfrak{u}}$, we restrict the attention in this section to $p>1$, i.e., $p^{*}<\infty$. We will use $[k+1: s]$ to denote

$$
[k+1: s]=\{k+1, k+2, \ldots, s\} \quad \text { or } \quad\{k+1, k+2, \ldots\} \text { if } s=\infty .
$$

Proposition 3 For POD weights and $k<s$, the truncation error is bounded by

$$
\left(\sum_{\mathfrak{u} \subseteq[k]} \frac{\gamma_{\mathfrak{u}}^{p^{*}}}{\left(\frac{q}{p^{*}}+1\right)^{p^{*}|\mathfrak{u}| / q}}\right)^{1 / p^{*}} \leq\left(\sum_{\mathfrak{v} \subseteq[k]} \frac{\gamma_{\mathfrak{v}}^{p^{*}}}{\left(\frac{q}{p^{*}}+1\right)^{p^{*}|\mathfrak{v}| / q}}\right)^{1 / p^{*}} T(k),
$$

where

$$
T(k)=\left(\sum_{l=1}^{s-k}\left(\frac{(l+k) !}{k !}\right)^{b p^{*}} \frac{1}{\left(\frac{q}{p^{*}}+1\right)^{p^{*} l / q}} \sum_{\substack{\mathfrak{w} \subseteq[k+1: s] \\|\mathfrak{w}|=l}} \prod_{j \in \mathfrak{w}} \gamma_{j}^{p^{*}}\right)^{1 / p^{*}} .
$$

Proof. Of course we have

$$
\begin{aligned}
\sum_{\mathfrak{u} \subseteq[k]} \frac{\gamma_{\mathfrak{u}}^{p^{*}}}{\left(\frac{q}{p^{*}}+1\right)^{p^{*}|\mathfrak{u}| / q}} & =\sum_{\mathfrak{v} \subseteq[k]} \sum_{\emptyset \neq \mathfrak{w} \subseteq[k+1: s]} \frac{\gamma_{\mathfrak{v} \cup \mathfrak{w}}^{p^{*}}}{\left(\frac{q}{p^{*}}+1\right)^{p^{*}(|\mathfrak{v}|+|\mathfrak{w}|) / q}} \\
& =\sum_{\mathfrak{v} \subseteq[k]} \frac{\gamma_{\mathfrak{v}}^{p^{*}}}{\left(\frac{q}{p^{*}}+1\right)^{p^{*}|\mathfrak{v}| / q}} T(\mathfrak{v}, k)^{p^{*}}
\end{aligned}
$$

where

$$
\begin{aligned}
& T(\mathfrak{v}, k)^{p^{*}}=\sum_{\emptyset \neq \mathfrak{w} \subseteq[k+1: s]}\left(\frac{(|\mathfrak{v}|+|\mathfrak{w}|) !}{|\mathfrak{v}| !}\right)^{b p^{*}} \frac{1}{\left(\frac{q}{p^{*}}+1\right)^{p^{*}|\mathfrak{w}| / q}} \prod_{j \in \mathfrak{w}} \gamma_{j}^{p^{*}} \\
& =\sum_{l=1}^{s-k}\left(\frac{(|\mathfrak{v}|+l) !}{|\mathfrak{v}| !}\right)^{b p^{*}} \frac{1}{\left(\frac{q}{p^{*}}+1\right)^{p^{*} l / q}} \sum_{\substack{\mathfrak{w} \subseteq[k+1: s] \\
|\mathfrak{w}|=l}} \prod_{j \in \mathfrak{w}} \gamma_{j}^{p^{*}} .
\end{aligned}
$$

Since $|\mathfrak{v}| \leq k$, we have

$$
\frac{(|\mathfrak{v}|+l) !}{|\mathfrak{v}| !} \leq \frac{(k+l) !}{k !} .
$$

This completes the proof. 
Example 2 Consider large $s$ and

$$
\gamma_{j}=\frac{c_{2}}{j^{a}} \quad \text { for } a>\max \left(1 / p^{*}, b\right) .
$$

Clearly

$$
\begin{aligned}
\sum_{\substack{\mathfrak{w} \subseteq[k+1: s] \\
|\mathfrak{w}|=l}} \prod_{j \in \mathfrak{w}} \gamma_{j}^{p^{*}} & =c_{2}^{l p^{*}} \sum_{j_{1}=k+1}^{s} j_{1}^{-a p^{*}} \sum_{j_{2}=j_{1}+1}^{s} j_{2}^{-a p^{*}} \ldots \sum_{j_{l}=j_{l-1}+1}^{s} j_{l}^{-a p^{*}} \\
& \leq c_{2}^{l p^{*}} \int_{k+1 / 2}^{\infty} x_{1}^{-a p^{*}} \int_{x_{1}}^{\infty} x_{2}^{-a p^{*}} \ldots \int_{x_{l-1}}^{\infty} x_{l}^{-a p^{*}} \mathrm{~d} x_{l} \ldots \mathrm{d} x_{2} \mathrm{~d} x_{1} \\
& =\left(\frac{c_{2}^{p^{*}}}{(k+1 / 2)^{a p^{*}-1}\left(a p^{*}-1\right)}\right)^{l} \frac{1}{l !} .
\end{aligned}
$$

Therefore

$$
T(k) \leq\left(\sum_{l=1}^{s-k}\left(\frac{(l+k) !}{k !}\right)^{b p^{*}} \frac{y^{l}}{l !}\right)^{1 / p^{*}}=\left(\sum_{l=1}^{s-k}((k+1) \cdots(l+k))^{b p^{*}} \frac{y^{l}}{l !}\right)^{1 / p^{*}}
$$

with

$$
y=\frac{c_{2}^{p^{*}}}{\left(\frac{q}{p^{*}}+1\right)^{p^{*} / q}\left(a p^{*}-1\right)(k+1 / 2)^{a p^{*}-1}} .
$$

Hence the upper bound in (10) can be computed efficiently using nested multiplication. We provide now the pseudo-code for doing that:

$$
\begin{aligned}
& y:=c_{2}^{p^{*}} /\left(\left(q / p^{*}+1\right)^{p^{*} / q}\left(a p^{*}-1\right)(k+1 / 2)^{a p^{*}-1}\right) \\
& T:=y s^{b p^{*}} /(s-k) \\
& \text { for } l=s-k-1 \text { to } 1 \text { step }-1 \text { do } \\
& \quad T:=(T+1)(l+k)^{b p^{*}} y / l \\
& \text { endfor } \\
& T:=T^{1 / p^{*}} .
\end{aligned}
$$

Furthermore, for $k \geq 2$,

$$
\begin{aligned}
& \left(\sum_{\mathfrak{v} \subseteq[k]} \frac{\gamma_{\mathfrak{v}}^{p^{*}}}{\left(\frac{q}{p^{*}}+1\right)^{p^{*}|\mathfrak{v}| / q}}\right)^{1 / p^{*}} \\
& \leq c_{1}\left(1+c_{2}^{p^{*}} \sum_{j=1}^{k} \frac{j^{-a p^{*}}}{\left(q / p^{*}+1\right)^{p^{*} / q}}+\sum_{\substack{\mathfrak{u} \subseteq[k] \\
|\mathfrak{u}| \geq 2}} \frac{(|\mathfrak{u}| !)^{b p^{*}} c_{2}^{|\mathfrak{u}| p^{*}}}{\left(q / p^{*}+1\right)^{p^{*}|\mathfrak{u}| / q}} \prod_{j \in \mathfrak{u}} j^{-a p^{*}}\right)^{1 / p^{*}}
\end{aligned}
$$

Now we provide an estimate for the last sum in this expression. We have

$$
\sum_{\substack{\mathfrak{u} \subseteq[k] \\|\mathfrak{u}| \geq 2}} \frac{(|\mathfrak{u}| !)^{b} p^{*} c_{2}^{|\mathfrak{u}| p^{*}}}{\left(q / p^{*}+1\right)^{p^{*}|\mathfrak{u}| / q}} \prod_{j \in \mathfrak{u}} j^{-a p^{*}}
$$




$$
\begin{aligned}
& =\sum_{\ell=2}^{k} \frac{(\ell !)^{b} p^{*} c_{2}^{\ell p^{*}}}{\left(q / p^{*}+1\right)^{p^{*} \ell / q}} \sum_{\substack{\mathfrak{u} \subseteq[k] \\
|\mathfrak{u}|=\ell}} \prod_{j \in \mathfrak{u}} j^{-a p^{*}} \\
& =\sum_{\ell=2}^{k} \frac{(\ell !)^{b p^{*}} c_{2}^{\ell p^{*}}}{\left(q / p^{*}+1\right)^{p^{*} \ell / q}} \sum_{\substack{\mathfrak{u} \subseteq[k] \\
|\mathfrak{u}|=\ell \\
\{1\} \subseteq \mathfrak{u}}} \prod_{j \in \mathfrak{u}} j^{-a p^{*}}+\sum_{\ell=2}^{k-1} \frac{(\ell !)^{b p^{*}} c_{2}^{\ell p^{*}}}{\left(q / p^{*}+1\right)^{p^{*} \ell / q}} \sum_{\substack{\mathfrak{u} \subseteq[2: k] \\
|\mathfrak{u}|=\ell}} \prod_{j \in \mathfrak{u}} j^{-a p^{*}} \\
& =\sum_{\ell=2}^{k} \frac{(\ell !)^{b} p^{*} c_{2}^{\ell p^{*}}}{\left(q / p^{*}+1\right)^{p^{*} \ell / q}} \sum_{\substack{\mathfrak{u} \subseteq[2: k] \\
|\mathfrak{u}|=\ell-1}} \prod_{j \in \mathfrak{u}} j^{-a p^{*}}+\sum_{\ell=2}^{k-1} \frac{(\ell !)^{b} p^{*} c_{2}^{\ell p^{*}}}{\left(q / p^{*}+1\right)^{p^{*} \ell / q}} \sum_{\substack{\mathfrak{u} \subseteq[2: k] \\
|\mathfrak{u}|=\ell}} \prod_{j \in \mathfrak{u}} j^{-a p^{*}} .
\end{aligned}
$$

For the two inner sums in the last expression we can now use the same method as in the derivation of (10) for the terms with indices $\ell=2, \ldots, k-1$, and hence obtain

$$
\begin{aligned}
\sum_{\substack{\mathfrak{u} \subseteq[k] \\
\mathfrak{u} \mid \geq 2}} \frac{(|\mathfrak{u}| !)^{b p^{*}} c_{2}^{|\mathfrak{u}| p^{*}}}{\left(q / p^{*}+1\right)^{p^{*}|\mathfrak{u}| / q}} \prod_{j \in \mathfrak{u}} j^{-a p^{*}} \\
\leq \sum_{\ell=2}^{k-1}(\ell !)^{b p^{*}-1}\left(\frac{c_{2}^{p^{*}}}{\left(q / p^{*}+1\right)^{p^{*} / q}\left(a p^{*}-1\right) 1.5^{a p^{*}-1}}\right)^{\ell}\left(\ell\left(a p^{*}-1\right) 1.5^{a p^{*}-1}+1\right) \\
\quad+\frac{(k !)^{b p^{*}-a p^{*}} c_{2}^{k p^{*}}}{\left(q / p^{*}+1\right)^{p^{*} k / q}}
\end{aligned}
$$

In analogy to product weights and using the upper bounds above, we calculated numbers $k=k(\varepsilon)$ which guarantee that

$$
\left(\sum_{\mathfrak{u} \nsubseteq[k]} \frac{\gamma_{\mathfrak{u}}^{p^{*}}}{\left(\frac{q}{p^{*}}+1\right)^{p^{*}|\mathfrak{u}| / q}}\right)^{1 / p^{*}} \leq \frac{\varepsilon}{2^{1 / p^{*}}}
$$

Since the upper bound (11) is not sharp for large $k$, i.e., small $\varepsilon$, we calculated the values of $k(\varepsilon)$ only for $a=4$. More precisely we did it for $s=10000, b=c_{1}=c_{2}=1, q=2, p=2$ and $p=\infty$, and $a=4$.

\begin{tabular}{c||c|c|c|c|c|c||l}
$\varepsilon$ & $10^{-1}$ & $10^{-2}$ & $10^{-3}$ & $10^{-4}$ & $10^{-5}$ & $10^{-6}$ & \\
\hline \hline$k(\varepsilon)$ & 3 & 8 & 26 & 81 & 256 & 809 & $p=\infty$ \\
\hline$k(\varepsilon)$ & 2 & 5 & 12 & 29 & 74 & 185 & $p=2$
\end{tabular}

\section{References}

[1] Gnewuch, M., Hefter, M., Hinrichs, A., Ritter, K., Wasilkowski, G.W.: On equivalence of weighted anchored and ANOVA spaces of functions with mixed smoothness of order one in weighted $L_{p}$ norms; unbounded domains. In preparation.

[2] Hefter, M., Ritter, K.: On embeddings of weighted tensor product Hilbert spaces. J. Complexity 31, 405-423 (2015). 
[3] Hefter, M., Ritter, K., Wasilkowski G.W.: On equivalence of weighted anchored and ANOVA spaces of functions with mixed smoothness of order one in $L_{1}$ and $L_{\infty}$ norms. J. Complexity 32, 1-19 (2016).

[4] Hinrichs, A., Schneider, J.: Equivalence of anchored and ANOVA spaces via interpolation. J. Complexity 33, 190-198 (2016).

[5] Kritzer, P., Pillichshammer, F., Wasilkowski G.W.: Very low truncation dimension for high dimensional integration under modest error demand. J. Complexity, to appear (2016). doi:10.1016/j.jco.2016.02.002

[6] Kritzer, P., Pillichshammer, F., Wasilkowski G.W.: On equivalence of anchored and ANOVA spaces; lower bounds. Submitted (2016).

[7] Kuo, F.Y., Schwab, C., Sloan, I.H.: Quasi-Monte Carlo finite element methods for a class of elliptic partial differential equations with random coefficients. SIAM J. Numer. Anal. 6, 3351-3374 (2012).

[8] Kuo, F.Y., Sloan, I.H., Wasilkowski, G.W., Woźniakowski, H.: On decompositions of multivariate functions. Math. Comp. 79, 953-966 (2010).

[9] Sloan, I.H., Woźniakowski, H.: When are quasi-Monte Carlo algorithms efficient for high dimensional integrals? J. Complexity 14, 1-33 (1998).

[10] Wasilkowski, G.W.: Tractability of approximation of $\infty$-variate functions with bounded mixed partial derivatives. J. Complexity 30, 325-346 (2014).

\section{Author's Address}

Peter Kritzer, Johann Radon Institute for Computational and Applied Mathematics (RICAM), Austrian Academy of Sciences, Altenbergerstr. 69, 4040 Linz, Austria.

Email: peter.kritzer(AT) oeaw.ac.at

Friedrich Pillichshammer, Department of Financial Mathematics and Applied Number Theory, Johannes Kepler University Linz, Altenbergerstr. 69, 4040 Linz, Austria.

Email: friedrich.pillichshammer(AT) jku.at

G.W. Wasilkowski, Computer Science Department, University of Kentucky, 301 David Marksbury Building, Lexington, KY 40506, USA.

Email: greg(AT)cs.uky.edu 